\title{
New experiment for WIMP direct search (NEWSdm)
}

\author{
Natalia Polukhina ${ }^{1,2,3}$, Nikolai Starkov ${ }^{1,2, *}$, on behalf of NEWSdm collaboration \\ ${ }^{1}$ Lebedev Physical Institute of RAS, 119991, Leninskii pr., 53, Moscow, Russia \\ ${ }^{2}$ National University of Science and Technology MISIS, 119049, Leninsky pr., 4, Moscow, Russia \\ ${ }^{3}$ National Research Nuclear University MEPhI, 115409, Kashirskoe Shosse, 31, Moscow, Russia;
}

\begin{abstract}
Dark Matter (DM) search is one of the most significant tasks of modern physics. Direct DM searches are nowadays one of the most fervid research topics with many experimental efforts devoted to the search for nucleus recoils induced by the scattering of Weakly Interactive Massive Particles (WIMP). The new experiment NEWSdm (Nuclear Emulsions for WIMP Search - directional measurement) aimed at direct search for DM particles is based on the technologies of nuclear emulsions combining high spatial resolution and the possibility of creating large mass detectors. Emulsion detectors able to reconstruct the direction of the nuclei recoiling on the WIMP are opening a new frontier to possibly extend DM searches beyond the neutrino background. The detector concept foresees the use of a bulk of nuclear emulsion surrounded with a shield from environmental radioactivity, to be placed on an equatorial telescope in order to cancel out the effect of the Earth rotation keeping the detector at a fixed orientation toward the expected direction of galactic WIMPs. Exploiting directionality would also prove the galactic origin of DM with an unambiguous signalto-background separation. The use in NEWSdm of fine-grained nuclear emulsions both as target and nanometric tracking device for directional DM searches gives a unique opportunity for a high-significance discovery of galactic DM.
\end{abstract}

\section{Introduction}

Compelling evidence for an abundant, non-baryonic, non-luminous dark matter component was accumulated over the past decades [1]. Nevertheless, the nature of the dark matter (DM) remains totally unknown, and the quest for an answer ranks as one of the main issues of the experimental particle physics, astrophysics and cosmology.

In the Standard Halo Model the Weakly Interacting Massive Particles (WIMP) [2,3] speed in the Galaxy is supposed to follow a Maxwellian distribution. If massive relics (WIMPs as theoretically appealing DM candidates) of the early Universe do exist, they are expected to be gravitationally bound to the baryonic visible matter. The motion of the Solar System through the Galaxy, however, should create an apparent wind of dark matter particles, blowing opposite to the direction of the Sun's motion towards the Cygnus constellation. The intensity of this wind, i.e. the WIMP flux, is expected to be timemodulated due to the Earth motion in the Solar System, with an annual period and a maximum rate in summer [4]. The origin of the DM annual modulation signature and of its peculiar features is due to the Earth motion with respect to the DM particles constituting the Galactic Dark Halo, so it is not related to terrestrial seasons. The speed of the Earth in the Solar System is anyway small compared to the speed of the Sun in the Milky Way, so the amplitude of the annual modulation is of the order of a few percent. The DAMA experiment [5] at LNGS has indeed reported a signal with a very clear evidence of annual modulation, as a possible indication of DM induced signal. This signal, although 
statistically extremely significant ( $>8$ standard deviations), is controversial because many experiments have already partially or totally excluded the region allowed by DAMA (see Fig. 1). Therefore DAMA results remain an intriguing puzzle.

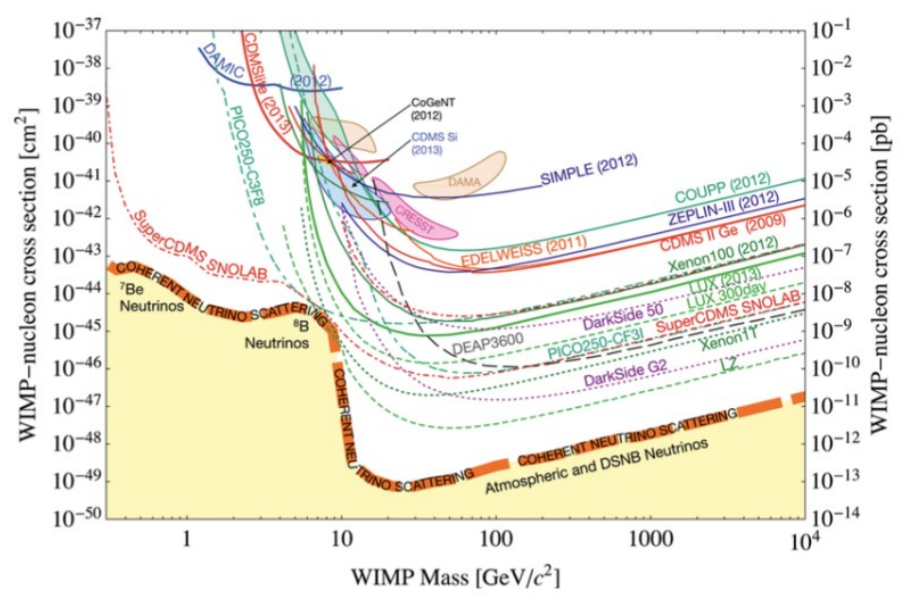

Fig. 1: Spin-independent WIMP-nucleon cross-section limits versus WIMP mass.

On the other hand, the angular distribution of the scattered nuclei is peaked around the direction of the apparent dark matter wind. The directional modulation is expected to be stronger than the annual modulation, with a rate of the forward-scattered nuclei one order of magnitude higher than of the backward-scattered ones. Since background sources are expected to be isotropic, the detection of a signal with a preferred direction would provide a powerful discrimination. Directional experiments intend to exploit this effect by measuring the direction of nucleus recoils, and hence the WIMP wind direction. A direct search for WIMPs in the mass range from a few $\mathrm{GeV} / \mathrm{c}^{2}$ to a few $\mathrm{TeV} / \mathrm{c}^{2}$ could be based on the detection of nucleus recoils induced by WIMP elastic scattering.

The NEWSdm project aims at the direct detection of dark matter candidates by measuring the direction of WIMP-induced nucleus recoils. For this challenge, the detector exploits nuclear emulsions of new generation with nanometric grains for an ultra-high spatial resolution. Key point is also absolutely new fully automated scanning systems capable to detect short tracks of 100-200 nm, with improved optical technologies overcoming the diffraction limit of conventional systems.

Given that nuclear emulsions are time insensitive, the detector will be placed on a standard equatorial telescope to keep its orientation fixed toward the Cygnus constellation. This experiment will demonstrate the potentiality of the technique and will start constraining the parameter space indicated by the DAMA experiment.

\section{Nano Imaging Tracker (NIT)}

Emulsion is used in particle physics since pion discovery at 1947 , and as for today, the most significant emulsion result was $v_{\mu} \rightarrow v_{\tau}$ oscillation discovery in appearance mode in the OPERA experiment [6]. Photographic nuclear emulsion is a suspension including lightsensitive microcrystals of silver halogenide distributed uniformly in gelatin [7]. Formation of a hidden image (i.e., of a track of the ionizing particle that can be chemically developed) in nuclear emulsion is due to the interaction of the charged particle with the electrons of atoms in the emulsion. When a charged particle passes through a crystal, its energy is 
absorbed in some of the emulsion grains by a bromide silver crystal, which brings to the formation of an aggregate of a certain number of silver atoms that can become the center of a hidden image. Thus, owing to its ionizing power, each charged particle penetrating emulsion leaves more or less thick track of exposed grains of silver halogenide. After the emulsion film development, these grains transform into metallic silver particles and become black (since they consist of finely divided crystalline aggregates). The chemical processing results in the track visible under a microscope as a chain of separate dots or as a solid line, depending on the particle charge. The chains of developed silver grains permit to reconstruct the particle trajectory with a significant precision (the angular measurement accuracy amounts to several milliradians). The size of a silver halide crystal in standard emulsion ranges from $0.1 \mu \mathrm{m}$ to $1 \mu \mathrm{m}$. The emulsion sensitivity strongly depends on the size of the crystals: the larger the grain, the higher the emulsion sensitivity to ionizing radiation.

Due to the low recoil energy of a WIMP-scattered nucleus, the expected track length is of the order of few hundred nanometers. State-of-the-art emulsions produced for the purposes of the OPERA experiment, with a linear dimension of the crystals of $200 \mathrm{~nm}$, are therefore not suitable for Dark Matter searches (fig.2). The novel emulsion films with grain diameters down to a few tens of nm produced in Nagoya University for NEWSdm are one order of magnitude smaller than conventional ones. These so-called Nano Imaging Trackers (NIT) and Ultra-Nano Imaging Trackers (U-NIT) have grains of 44 and $18 \mathrm{~nm}$ diameter respectively. The NIT films have a linear density of crystals of about 11 crystals $/ \mu \mathrm{m}$, while U-NIT show 29 crystals/ $\mu \mathrm{m}$. They make it possible to reconstruct trajectories with lengths shorter than $100 \mathrm{~nm}$, if analyzed by means of microscopes with enough resolution. So, micronization of crystals is a key for detection short tracks of nucleus recoils from WIMPs (fig.2).

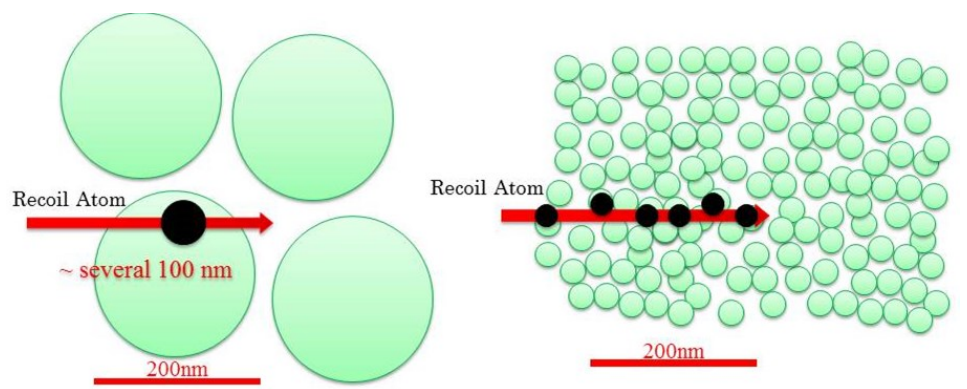

Fig.2 Illustration of the elementary particles latent image production in different emulsion types: OPERA emulsion with $\mathrm{AgBr}$ crystal size $\sim 200 \mathrm{~nm}$ (left); NIT emulsion with $\mathrm{AgBr}$ crystal size $\sim 40 \mathrm{~nm}$ (right)

Each kind of nuclear emulsion includes about 10 basic chemical elements. In addition to heavy elements as silver and bromine, nuclear emulsions also containe lighter ones, such as $\mathrm{C}, \mathrm{N}, \mathrm{O}$ and $\mathrm{H}$. It makes nuclear emulsion sensitive to a wide range of possible WIMP masses, as WIMPs can dissipate on any of these elements. Heavy WIMPs $(>50 \mathrm{GeV})$ will most effectively be scattered on $\mathrm{Ag}$ and $\mathrm{Br}$, light ones $(5-50 \mathrm{GeV}$ ) - on $\mathrm{C}, \mathrm{N}, \mathrm{O}$, and ultralight - on $\mathrm{H}$. Each element gives a specific contribution to the overall sensitivity, and the lighter nuclei give longer range at the same recoil energy (fig.3). 


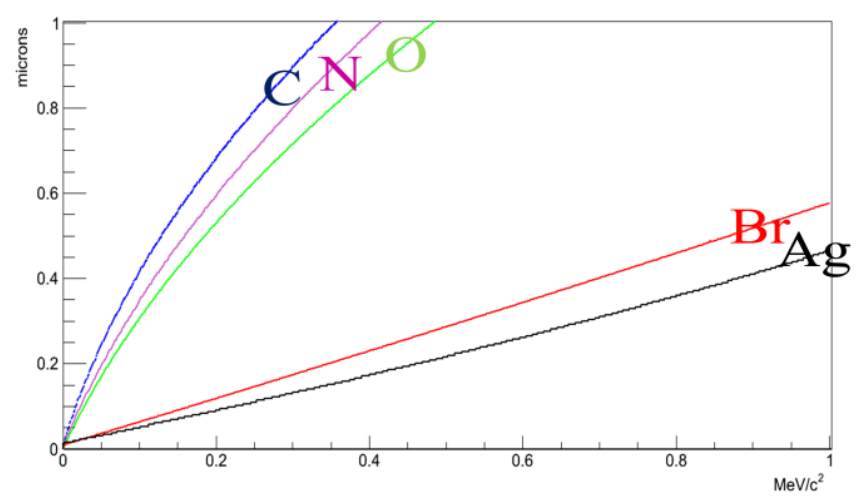

Fig.3 Dependence of the track length from recoil nuclei energy for different elements of emulsion composition.

In addition to the above advantages, the nuclear emulsion has got high spatial resolution, allowing direct observations of the recoil nuclei direction. The novel emulsions will detect tracks with lengths of about $100 \mathrm{~nm}$, what is not available to other existing solidstate or liquid detectors. In gases, because of their low density, the recoil nucleus path is long enough to register a track, but the measurement accuracy of the gas detectors is strongly limited by diffusion effects, which lead to their low sensitivity to the WIMPs. In contrast, the high density emulsions provide enough sensitivity for WIMP registration.

The experiment under development will be based primarily on the emulsion ability of the precise determination of the recoil nucleus direction. The detector is planned to be placed on a moving platform (fig.4), which will be oriented to the Cygnus constellation during the entire exposure time, so that the WIMPs will always arrive in the detector from one direction, while the directions to possible background sources will vary. In this case, the anisotropy of the signal can be interpreted as presence of the "wind" of particles from the Cygnus constellation.

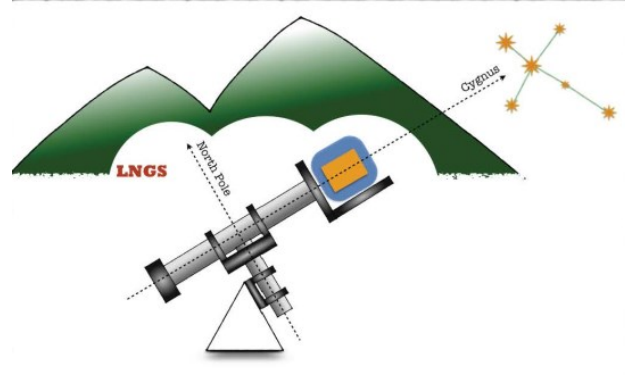

Fig.4 Scheme of the NEWSdm detector installation.

According to astrophysical models, our Galaxy is surrounded by halo of dark matter particles which due to its weak interaction with the ordinary matter revolves around the common center of mass regardless of it. Generally speaking, the local velocity of the halo particles in the region of the Solar System does not match with the velocity of the latter, which leads to formation of the dark matter particle "wind". Now, the Solar System moves towards the Cygnus constellation, so it seems that the "wind" is blowing from there.

\section{Readout system}


In the NEWSdm experiment the expected WIMP signal will consist of short-path, anisotropically distributed, nuclear recoils over anisotropically distributed background. The search for signal candidates requires the scanning of the whole emulsion volume. The readout system has therefore to fulfill as main requirements: a fast, completely automated, scanning system is needed to analyze the target volume over a time scale comparable with the exposure; the spatial resolution has to be improved by more than one order of magnitude compared to that achieved with standard emulsion films, reaching the challenging value of a few tens of nanometers, in order to ensure high efficiency and purity in the selection of signal candidates.

The signal confirmation will be obtained with powerful optical microscope equipped with a light polarizer: exploiting the different response of non spherical grain clusters to different polarization angles, the unprecedented spatial resolution of $10 \mathrm{~nm}$ is obtained. The idea of using the plasmon resonance effects is a base for new high resolution automatis microscope. Such resonance appears on the surface of silver grains under their illumination (fig. 5). This resolution allows to measure track length shorter than one hundred nanometers providing a very high signal to noise ratio.
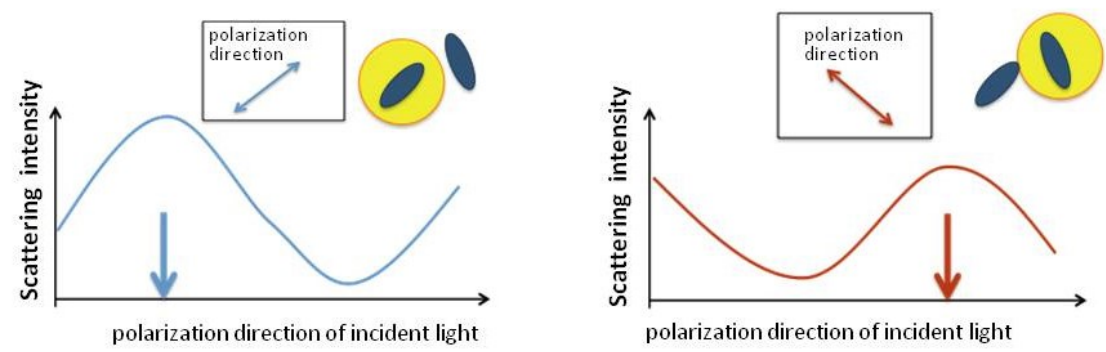

Fig.5 Taking multiple measurements over the whole polarization range produces a displacement of the barycenter of the cluster

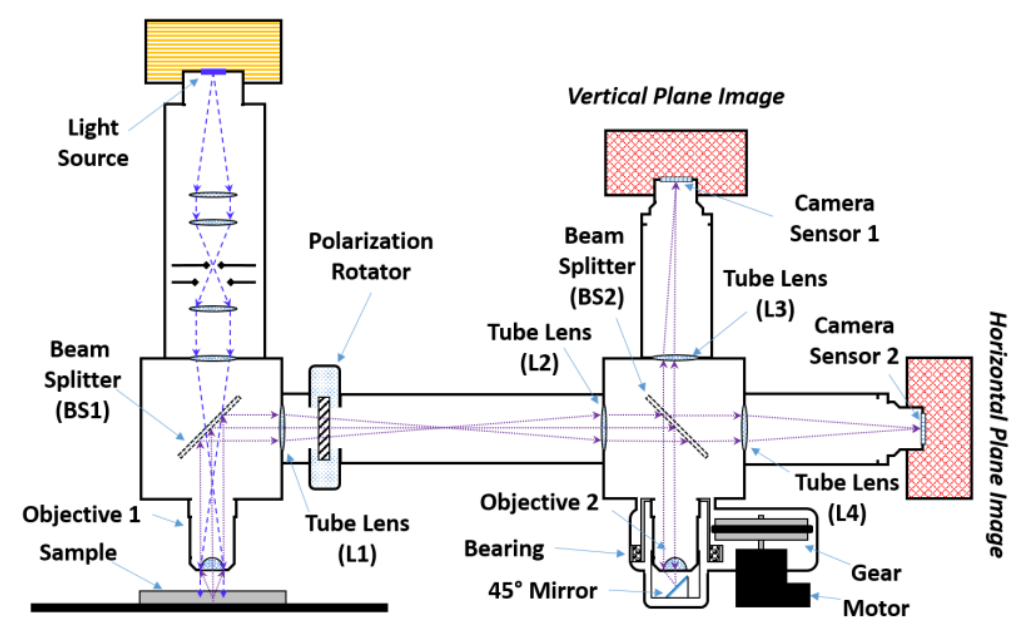

Fig.6 The scheme of 3D nanometric readout for NEWSdm

The scanning method consists of taking a series of tomographic images while moving the focal plane of the objective inside the sensitive emulsion layer and in this way digitizing the full content of the sample. After the image processing and the three-dimensional reconstruction, one obtains shapes and positions of all silver grains. Images are taken with 
steps shorter than the objective's depth of field: in this way the digitization and analysis of each cubic micron of sample is performed without gaps. The huge amount of information (up to several GB/s) coming from fast digital cameras requires high performance hardware, together with advanced image processing and computing algorithms. The image taking is synchronized with the motion of the microscope motorized stage and the objective lens (XYZ axes).

Plasmon analysis performing in horizontal plane (fig.6) include measuring $\phi$ angle, mirror rotation to make the vertical plane coincide with the prediction's direction $(\phi)$. Plasmon analysis performing in vertical plane include measuring $\theta$ angle and measuring $3 \mathrm{D}$ track length.

The starting point of the emulsion scanning is the image analysis to collect clusters made of dark grains at several depths across the emulsion plate thickness. A cluster made of several grains indeed tends to have an elliptical shape with the major axis coincident with the direction of the trajectory, while a cluster produced by a single grain tends to have a spherical shape. NEWSdm use plasmon resonance technology to retrieve track information in NIT emulsion beyond the optical resolution.

\section{Conclusion and perspectives}

So, the NEWSdm aim is to detect the direction of the nucleus recoils produced in WIMPs interactions. Nanometric nuclear emulsions acting both as target and tracking detector use in experimental setup. Location in underground Gran Sasso Laboratory and neutron shield surrounding the target allow to realize the background reduction. The detector concept foresees the use of a bulk of nuclear emulsion surrounded with a shield from environmental radioactivity, to be placed on an equatorial telescope in order to cancel out the effect of the Earth rotation keeping the detector at a fixed orientation toward the expected direction of galactic WIMPs. Exploiting directionality would also prove the galactic origin of DM with an unambiguous signal-to-background separation.

In March 2017 the NEWSdm Collaboration has installed an experimental setup for the exposure of first $\sim 10 \mathrm{~g}$ detector at the Gran Sasso INFN Underground Laboratories. This test was aim at measuring the detectable background from environmental and intrinsic sources and to validate estimates from measurements [8] and simulations. The confirmation of a negligible background will pave the way for the construction of a pilot experiment with an exposure on the $\sim 10 \mathrm{~kg}$ year scale. This pilot experiment acted as a demonstrator to further extend the sensitivity towards the neutrino floor.

Due to analysis of first test exposure it is possible to say that key points and plans towards an experiment are next:

- Signal:

- capability to see nano-tracks demonstrated;

- Need to measure track parameters;

- Important calibration with SEM;

- Improve optical microscopy: colour camera, fast scanning;

- Improve sensitivity by using lower size grains, enough optical contrast;

- Background reduction:

- Fog;

- electron recoils from ${ }^{14} \mathrm{C} \sim 10^{8} / \mathrm{kg} /$ year reqiure rejection power for $10 \mathrm{~g} \mathrm{x}$ 1 month $>10^{5} \div 10^{6}$. In perspective $\left(\sim \mathrm{kg}\right.$ year) requires $\sim 10^{8} \div 10^{9}$ and more;

- Low temperature conditions $\left(-80^{\circ} \mathrm{C}\right)$, check electron sensitivity at low temperature;

- Use machine learning technique for signal/background discrimination;

- Emulsion production underground in a clean room; 
- Design appropriate shield with equatorial telescope.

So, conclusion is that nuclear emulsions with nanometric grains open the way for a directional dark matter search with high sensitivity. NEWSdm collaboration realized breakthrough in readout technologies for optical microscopes (no need for X-ray confirmation (much faster and convenient), and push the track length threshold down (higher sensitivity)). First test exposure confirmed results of model calculation and demonstrated that neutron background from intrinsic radioactivity negligible up to $\sim 10 \mathrm{~kg}$ year. NEWSdm plan to prepare a kg scale experiment as a demonstrator of the technology and the first spin-independent search of this kind.

\section{References}

1. K.A. Olive et al. (Particle Data Group), Chin. Phys. C, 38, 090001 (2014)

2. G. Bertone, Particle Dark Matter, Cambridge University Press, (2010);

3. M. W. Goodman and E. Witten, Phys. Rev. D 31 (1985) 3059

4. D. N. Spergel, Phys. Rev. D 37 (1988) 1353

5. https://arxiv.org/pdf/1805.10486.pdf

6. N. Agafonova et al. (OPERA Collaboration), Phys.Rev.Lett. 115 (2015) 12, 121802

7. Powell C F, Fowler P H, Perkins D H The Study of Elementary Particles by the Photographic Method: An Account of the Principal Techniques and Discoveries, Illustrated by an Atlas of Photomicrographs (London: Pergamon Press, 1959)

8. A. Alexandrov et al., Astroparticle Physics 80 (2016) 16 